\title{
Step energy and step interactions on the reconstructed GaAs(001) surface
}

\author{
Rita Magri, ${ }^{*}$ Sanjeev K. Gupta, ${ }^{\dagger}$ and Marcello Rosini ${ }^{\ddagger}$ \\ Dipartimento di Fisica, Informatica e Matematica (FIM) dell'universitá degli studi di Modena e Reggio Emilia and S3 research center of \\ CNR-INFM, via Campi 213/A, 41100 Modena, Italy
}

(Received 19 February 2014; revised manuscript received 22 July 2014; published 30 September 2014)

\begin{abstract}
Using $a b$ initio total energy calculations we have studied the relation between the step atomic configuration and its properties (step energy, donor/acceptor behavior, and step interaction) on a $\beta_{2}(2 \times 4)$ reconstructed GaAs (001) surface. The results have been tested against the widely used elastic dipole model for the step energy and step interaction considered valid for stress-free surfaces. We have found that acceptor-behaving steps have an attractive interaction and donor-behaving steps have a repulsive interaction in contrast with the elastic dipole model which predicts always a repulsive interaction between like-oriented steps. To account for the attractive interaction we consider the electrostatic dipole interaction having the $L^{-2}$ scaling with the step distance $L$ and therefore compatible with the standard elastic model. Using a model charge distribution with localized point charges at the step based on the electron counting model we show that the electrostatic step interaction can indeed be generally attractive and of the same order of magnitude of the negative elastic dipole interaction. Our results show however that the usually employed dipole model is unable to account for the repulsive/attractive step interaction between donorlike/acceptorlike steps. Therefore, the ab initio results suggest an important electronic contribution to the step interaction, at least at the short step distances accessible to the first-principles study. Our results explain qualitatively many experimental observations and provide an explanation to the step bunching phenomenon on $\operatorname{GaAs}(001)$ induced by doping or by critical growth conditions as due to the stabilization of attractively interacting step structures. These ideas would lead to the development of a bottom-up surface step engineering.
\end{abstract}

DOI: 10.1103/PhysRevB.90.115314

PACS number(s): 68.35.B-, 68.47.Jn, 71.15.Mb, 71.15.Nc

\section{INTRODUCTION}

Extended surface defects such as surface steps play a crucial role in epitaxial growth, deciding the growth mode [1], the favorable sites for island nucleation [2], the island shape and evolution, roughening and facetting, and structure and stability of vicinal surfaces. Despite their importance most theoretical investigations have addressed so far steps on metal or silicon surfaces [3]. However, the progress in semiconductor homoand heteroepitaxy, leading to spontaneous self-assembly of semiconductor and metal nanostructures on semiconductor surfaces [1], requires a better understanding of step stability and dynamics to assess precisely their role in the epitaxial growth of interfaces and nanostructures. To study the stability and atomic structure of steps and vicinal surfaces, and the relation between their structure and properties is a first step in this direction. Here we address the III-V (001) technologically important surfaces. Unfortunately these surfaces show complex ambient-dependent reconstructions making the problem very difficult to tackle. Few semiconductor surface steps were studied in some detail, among them the stepped $(1 \times 2) /(2 \times 1) \mathrm{Si}(001)$ surface [4-7] and $\mathrm{Si}(111): \mathrm{H}$ surface $[8,9]$ using atomistic (semiempirical or $a b$ initio) or continuous theory methods. GaAs(001) surface, on the other hand, forms reconstructions having a much larger periodicity and structural complexity. Because of the inherent large

\footnotetext{
*rita.magri@unimore.it

†Present address: Department of Physics, St. Xavier's College, Navrangpura, Ahmedabad 380009, India.

${ }^{\ddagger}$ Permanent address: Margen, Via Dino Ferrari, Maranello (MO), Italy.
}

dimensions of the surface unit cells required to describe stepped reconstructed semiconductor surfaces, few atomistic calculations [10] and even less ab initio calculations have been attempted [11].

Stability of stepped surfaces is usually described in terms of the step energy, which is the energy required to form a single step line on a flat surface, and the step-step interaction. At $T=0 \mathrm{~K}$ steps are free of kinks and for surfaces free of external stresses, such as the lateral strains imposed by growth on mismatched substrates or surface stress anisotropies caused by broken symmetry domains [5,7], the step-step interaction is commonly described using the Marchenko and Parshin theory (MP) [12] where steps are modeled by periodic lines of point force dipoles on otherwise perfectly flat surfaces. Within this theory the step-step elastic interaction energy scales as $L^{-2}$ with the step distance $L$ and is repulsive for like-oriented steps.

$\mathrm{GaAs}(001)$ step structure and vicinal surfaces to $\mathrm{GaAs}(001)$ have been the object of experimental investigations [1315] that have not found adequate support by theoretical or computational works. In As-rich conditions GaAs(001) shows two main reconstructions: $c(4 \times 4)$ at lower growth temperatures and $\beta_{2}(2 \times 4)$ at higher temperatures, the transition temperature depending strongly on the As/Ga flux ratio. Experiments have shown that the thinnest steps on GaAs(001) $\beta_{2}(2 \times 4)$ reconstructed surfaces are two atomic layers high, since the last layer is always As terminated. Thus GaAs(001) steps correspond to the double layer steps on $\mathrm{Si}$ (001) and are essentially of two kinds: steps oriented along [110] [A steps, corresponding to the DB steps of $\mathrm{Si}(001)]$ or along [110] [B steps, corresponding to the DA steps of $\mathrm{Si}(001)]$. For the $\beta_{2}(2 \times 4)$ reconstructed surfaces A steps run parallel to the As dimer bond direction, while B steps run orthogonal to them. 
Heller et al. [13] extracted an estimate of the step energies of A and $\mathrm{B}$ steps counting the kinks on $\beta_{2}(2 \times 4)$ surfaces grown in quasiequilibrium conditions under the assumption that kinks form in a uncorrelated way and found that the step energy of B steps is about 6 times [13] or 10 times [14] higher than that of $\mathrm{A}$ steps. As a consequence, B steps are rougher than A steps and growing islands are elongated along the [110] surface direction which is the A step direction. As for the step-step interaction the constant $K$ of the MP repulsive $K L^{-2}$ interaction between steps at distance $L$ was estimated by analyzing the terrace width distributions and correlation lengths for a sequence of steps in thermodynamic equilibrium [15]. The authors found for A steps a very large value of $K: K=20-30 \mathrm{eV}$ or $K=1.5$ $\mathrm{eV}$ using theirs or Heller's [13] data, respectively, whereas the interaction between B steps was found to be much weaker. The very large $K$ of A steps was explained speculating that the interaction constant is dominated not by the elastic interaction but by a strong repulsive electric dipole interaction [15]. In these experiments the surface reconstruction was reported to be $\beta_{2}(2 \times 4)$.

In this paper we extract the step energy and the step-step interaction from sets of first-principles calculations on vicinal surfaces to the $\beta_{2}(2 \times 4)$ reconstructed $\mathrm{GaAs}(001)$ at different miscut angles, misoriented towards (111) (A steps) or (111) (B steps). We have found that A steps of monoloyer height are more stable than the equally high B steps in agreement with the experiment. B steps have a weaker step interaction than A steps also in agreement with the experiment. Interestingly, A steps have both short-range repulsive or attractive step interactions depending on their atomic structure. In particular, As-rich steps behaving as acceptor defects have an attractive interaction, while As-poor steps behaving as donor defects have a repulsive interaction. A "donorlike" step becomes stable at relatively large distances and is likely to be formed at low temperatures. However, the unstable attractively interacting As-rich steps could form when stabilized by donor impurities such as silicon or As-rich and high temperature growth conditions. Since attractive interactions can be at the origin of the formation of step bunching our results could explain the observed formation of step bunches when the samples are in those conditions [15,16]. The attractive step interaction can be explained accounting for the electrostatic point-dipole model. An estimate of the electrostatic point-dipole interaction using the electron counting rule (ECR) assuming localized dangling bond charges at the steps reveals that the interaction is indeed generally attractive.

\section{METHOD}

The vicinal surfaces are modeled through a sequence of monolayer or bilayer-high equally spaced steps oriented along the [110] (A steps) or [1110] (B steps) surface directions, respectively. Different step configurations are considered that are compatible with STM observations [13]. The atomic structural models of A steps are shown in Fig. 1. Seven different atomistic models for A and B step geometries, denoted $a$ to $g$, are studied. These atomic configurations correspond to different ways the $\beta_{2}$ reconstruction can be matched at the up and down ledges. For each A step configuration shown in Fig. 1, further structures are obtained shifting the single or double As dimers up and down of $a / 2$ along the [110] direction, across the step, where $a$ is the surface lattice constant, $a=a_{0} / \sqrt{2}$, with $a_{0}$ the GaAs lattice constant.

We have calculated the surface energy $\gamma$ of the vicinal surfaces and compared it to that of the unstepped $\beta_{2}(2 \times 4)$ surface. The calculations are performed in the framework of the plane-wave density functional theory (DFT) and the local density approximation (LDA) using the open source package QUANTUM ESPRESSO (http://www.quantum-espresso.org) [17]. The surfaces are modeled through repeated slabs along the [001] direction. The calculations are carried out in a supercell geometry with periodic boundary conditions. The supercell dimension for A steps is $2 a$ along the [1110] direction and ranges from $4.5 a$ to $17.5 a$ along the [110] direction. For B steps the surface unit cell is $4 a$ along the [110] direction and ranges from to $5.5 a$ to $11.5 a$ along the [110] direction. The atoms at the bottom layer of the slab are kept fixed at the theoretical bulk positions, and their dangling bonds are passivated with pseudohydrogen atoms of fractional charge, in order to mimic the constraint due to the semi-infinite bulk. The remaining atoms have been relaxed until forces were less than $0.005 \mathrm{eV} / \AA$. The slab is 13 atomic planes thick for A steps and 12 atomic planes thick for B steps excluding the pseudohydrogen plane. The slabs are separated by a vacuum region of about $15 \AA$ in order to minimize the interactions across the boundaries. The As and Ga pseudopotentials for the $s$ and $p$ valence electrons are norm conserving, separable, and core corrected and the plane wave energy cutoff is 15 Ry which yielded structural and elastic parameters for the metal elements and bulk GaAs in good agreement with the experimental values [18]. The convergence of the calculated $\gamma$ on the Brillouin zone sampling has been accurately tested finding that the sampling along the $[\overline{1} 10]$ direction is crucial. To obtain good converged results (within $0.3 \mathrm{meV} / \AA$ ) the grid needs to be dense (at least eight $k$ points) along this direction. For this reason we used a grid of $16 k$ points to sample the Brillouin zone. A small metallic smearing $(0.26 \mathrm{eV})$ was used to account for the metallicity of the step configurations. The contribution of the hydrogenated backside has been subtracted using similarly calculated energies of stepped slabs of analogous dimensions, hydrogenated on both sides. The procedure we employ for the subtraction is schematically shown in Fig. 2. In Fig. 2(a) the supercell of the vicinal surface having steps $\mathrm{A} a$ separated by two $\beta_{2}$ terraces is shown (replicated twice along the direction orthogonal to the step line, i.e., the [110] direction, $x$ direction). The hydrogenated backside (delimited by the dashed rectangle in the figure) energy contribution is subtracted by calculating also the total energies of the systems (b) and (c) depicted in Figs. 2(b) and $2(\mathrm{c})$, respectively, and using $E_{\text {vicinal }}=E_{(a)}-E_{(b)}+\frac{1}{2} E_{(c)}$, where $E_{(a)}, E_{(b)}$, and $E_{(c)}$ are the total energies of the three systems (a), (b), and (c) in Fig. 2. The total energies of the three structures are calculated using equivalent $k$-point meshes. This procedure is followed for all the structures calculated in this work. Further details about the calculations are given elsewhere [11].

To derive the surface energies $\gamma$ of vicinal surfaces with step termination $i$ and miscut angle $\alpha$ at temperature $T=0 \mathrm{~K}$ 


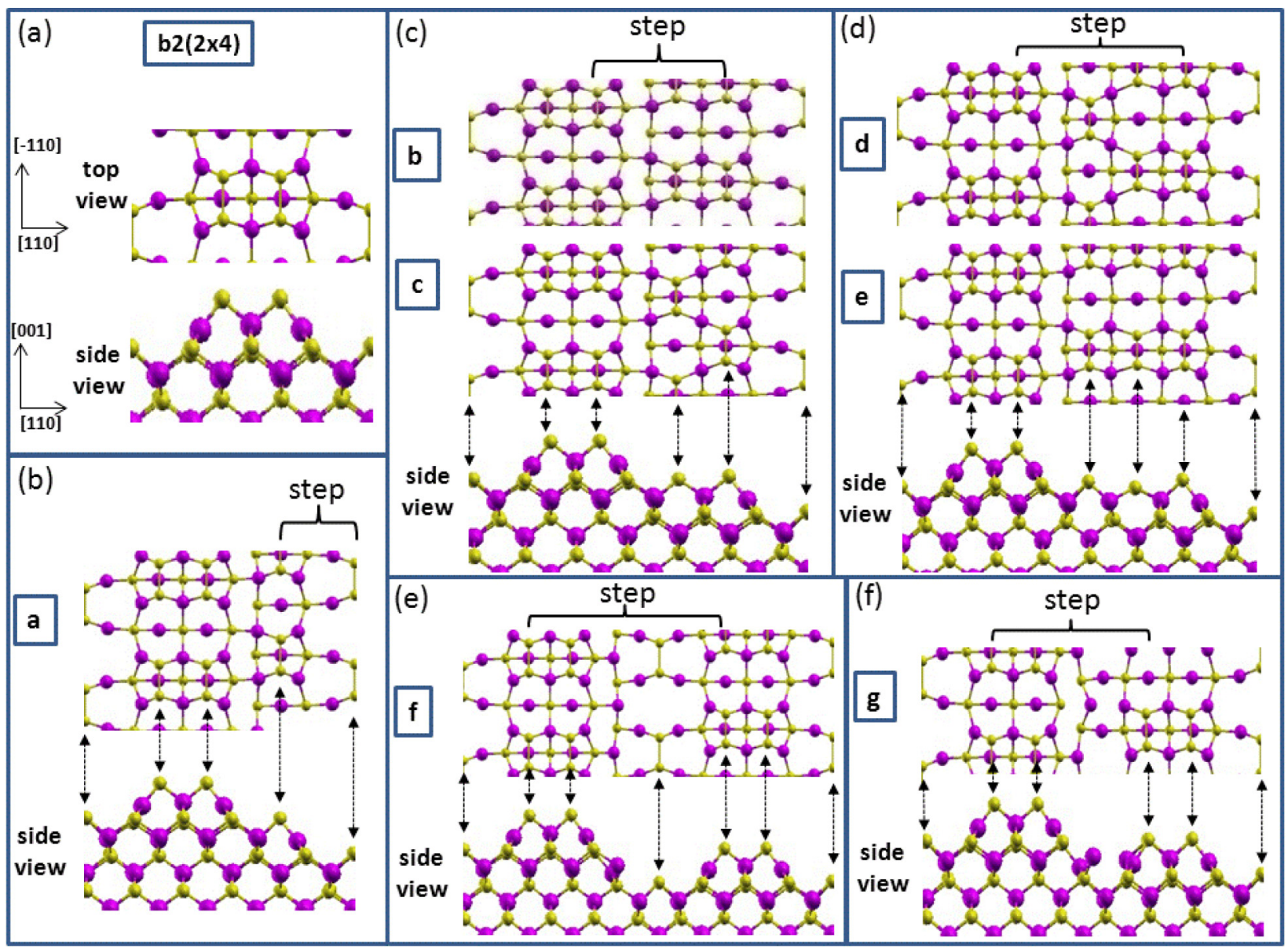

FIG. 1. (Color online) Top and side views of A steps with terraces only one $\beta_{2}$ unit cell long: (a) the $\beta_{2}$ structure, (b) the step A $a$, (c) the steps $\mathrm{A} b$ and $\mathrm{A} c$, (d) the steps $\mathrm{A} d$ and $\mathrm{A} e$, (e) the step $\mathrm{A} f$, and (f) the step $\mathrm{A} g$. In the figure are shown the unit cells of the shortest step structures of each kind. The vertical double arrows relate the top view and the side view indicating the position of the surface As dimers. The step part of the unit cells has been indicated for each structure. Purple balls: Ga atoms; yellow balls: As atoms.

we use the expression

$$
\begin{aligned}
\gamma_{i, \alpha}\left(\mu_{\mathrm{As}}\right)= & \frac{\left(E_{i, \alpha}-n_{\mathrm{Ga}} \mu_{\mathrm{GaAs}}^{\mathrm{bulk}}+\left(n_{\mathrm{Ga}}-n_{\mathrm{As}}\right) \mu_{\mathrm{As}}^{\mathrm{bulk}}\right)}{S} \\
& +\frac{\left(n_{\mathrm{Ga}}-n_{\mathrm{As}}\right) \Delta \mu_{\mathrm{As}}}{S}, \\
= & \gamma_{i, \alpha}\left(\Delta n_{i}=0\right)+\frac{\Delta n_{i}}{S}\left(\mu_{\mathrm{As}}^{\mathrm{bulk}}+\Delta \mu_{\mathrm{As}}\right),
\end{aligned}
$$

where $E_{i, \alpha}$ is the vicinal surface energy. The label $i$ refers to the specific step atomic configuration, $i=a, \ldots, g$ (see Fig. 1), and $\alpha$ is the miscut angle, $\tan (\alpha)=h / L, h$ being the step height ( 1 or $2 \mathrm{ML}$ ) and $L$ the terrace length. $S$ is the surface unit cell area and $\mu_{\mathrm{GaAs}}^{\text {bulk }}$ the formation energy of one Ga-As pair in bulk GaAs. $n_{\mathrm{Ga}}$ and $n_{\mathrm{As}}$ are the number of Ga and As atoms in the system. $\Delta n_{i}=n_{\mathrm{Ga}}-n_{\mathrm{As}}$ is the surface with step $i$ stoichiometry. $\Delta n=2$ is the $\beta_{2}$ stoichiometry. Thus $\Delta n_{\text {step }}^{i}=$ $\Delta n_{i}-\Delta n R_{S, i}$, where $R_{S, i}$ is the ratio between the step and $\beta_{2}$ surface areas, defines the isolated step stoichiometry. The surface energy depends on the growth conditions via the Ga and As chemical potentials. This dependency is expressed in Eq. (1) by the deviation of the As chemical potential $\Delta \mu_{\text {As }}$ (treated as a variable quantity) from the value it has in the bulk rhombohedral As metal (e.g., $\Delta \mu_{\mathrm{As}}=0$ for $\mu_{\mathrm{As}}=\mu_{\mathrm{As}}^{\mathrm{bulk}}$ ).
We have found that for A steps the surface energy change related to the in-plane shifts of (single-single, single-double, and double-double) As dimers parallel to the step $i$ direction (due to the degeneracy of the As dimer position) is an order of magnitude smaller $\left(<0.05 \mathrm{meV} / \AA^{2}\right.$ for $\left.\tan \alpha>0.1\right)$ than the energy difference between the step structures $i$ ( $a$ to $g$ ), so we next consider only the actual steps $i$ as shown in Fig. 1. In Fig. 3 the reduced projected surface energies $\left(\gamma^{\prime}=\gamma / \cos \alpha\right)$ [19] of vicinal surfaces formed by A and B steps, respectively, are plotted as a function of $\tan \alpha$ for two values of the As chemical potential at the end points of the calculated range of stability of the $\beta_{2}(2 \times 4)$ [and the slightly more stable $c(2 \times 8)$ ] reconstruction.

To extract the step properties the reduced surface energies are fit to the relation [20]

$\gamma_{i}^{\prime}\left(\alpha, \mu_{\mathrm{As}}\right)=\gamma\left(0, \mu_{\mathrm{As}}\right)+\frac{\epsilon_{i}\left(\mu_{\mathrm{As}}\right)}{h} \tan \alpha+q_{i}\left(\mu_{\mathrm{As}}\right)(\tan \alpha)^{3}$,

where $\gamma\left(0, \mu_{\mathrm{As}}\right)$ is the $\beta_{2}(2 \times 4)$ (miscut $\alpha=0$ ) surface energy, $\epsilon_{i}$ is the step energy, $\epsilon_{i}=h\left(d \gamma_{i} / d \alpha\right)_{\alpha=0}$, i.e., the energy per unit step length of a single isolated step of structure $i$, and $q_{i}\left(\mu_{\mathrm{As}}\right)$ is the contribution of the step-step interaction. 


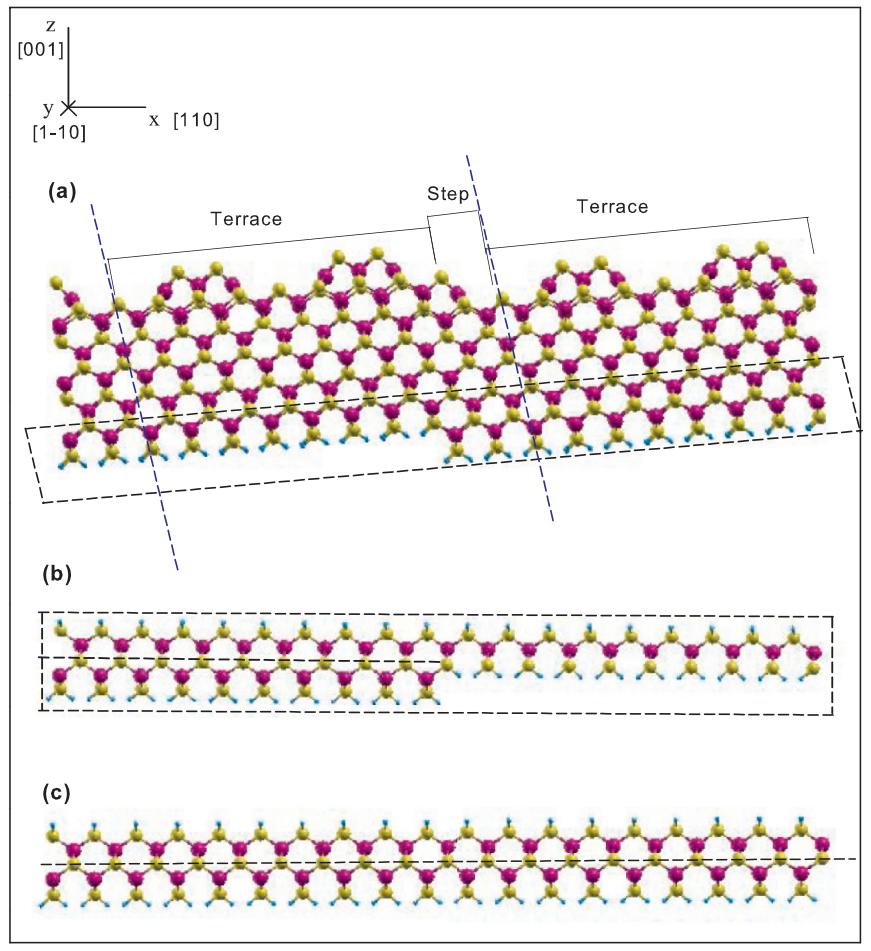

FIG. 2. (Color online) (a) Ball and stick model of the slab featuring the vicinal surface having steps of kind $\mathrm{A} a$. The terrace (two $\beta_{2}$ unit cell long) and step regions along the [110] direction are shown. The unit cell dimension along the [110] direction is comprised between the two dashed blue lines. (b) The hydrogenated back side of the slab. (c) The hydrogenated flat slab used to subtract the energy of the top side of the structure (b). Yellow balls represent arsenic atoms, purple balls represent gallium atoms, and cyan small balls represent pseudohydrogen atoms.

\section{RESULTS}

\section{A. Step energy}

The results of the fits are reported in Fig. 3 as solid lines. Equation (2) has been used to interpret STM images of stepped surfaces to extract the step parameters in the case of Si and Ge (001) surfaces [21]. The step energy $\epsilon_{i}$ is generally considered to be positive since the formation of a step goes along with the creation of additional dangling bonds (DBs). The last term in Eq. (2) derives from the assumption that the step-step interaction exhibits a $L^{-2}$ decay. This decay was derived, within isotropic continuous elasticity, for the elastic field interaction between force dipoles localized at $\delta$-like positions on a flat surface [12]. The model predicts repulsive interactions between like-oriented steps and attractive interactions between opposite-oriented steps. Equation (2) has been used to fit empirically calculated data of single and double step energetics on the $\mathrm{Si}(001)$ surface [7]. Generally dipolar long-range step-step interactions were shown [22] to decay to the lowest order as $L^{-2}$.

The step energies relative to different $\Delta \mu_{\mathrm{As}}$ are given in Table I. We can see that B steps (all one ML high) have a higher formation energy than the one ML high A steps in agreement with the experiments $[13,14]$ by Heller et al. discussed in the Introduction section.
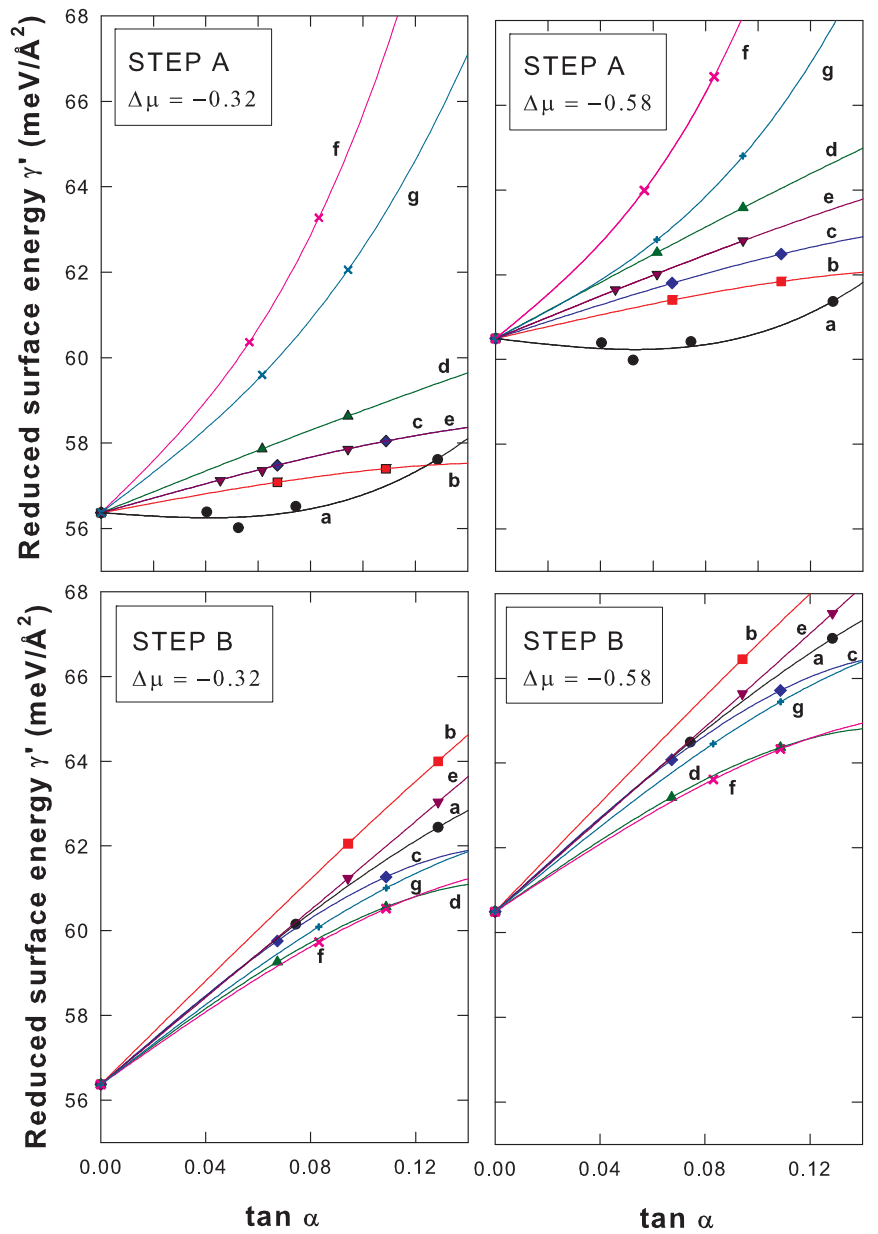

FIG. 3. (Color online) Reduced surface energies versus miscut angles $\tan \alpha$ for steps $\mathrm{A}$ and $\mathrm{B}$ at $\Delta \mu_{\mathrm{As}}=-0.32$ and $\Delta \mu_{\mathrm{As}}=-0.58$.

We focus now on the more stable A steps. The step energy is roughly related to the number of additional DBs $N_{\mathrm{DB}}$ inserted with the step $[23,24]$ modified by the effect of the As chemical potential via the step stoichiometry $\Delta n_{\text {step }}^{i}$, which changes the degree of each step (un)stability depending on the external conditions. We find that steps $\mathrm{A} a$ have a negative step energy (respective to the $\beta_{2}$ "flat" surface) and become stable when sufficiently far apart. Negative step energies were calculated also for SB and DB steps on the $2 \times 1 \mathrm{Si}(001)$ using atomistic interatomic potentials [7]. For these steps the destabilizing effect due to the additional dangling bonds introduced by the step is largely offset by an additional release of the surface elastic stress. Indeed, while reconstructions lower the surface energy by creating new bonds between the atoms at the surface (formation of dimers, for example), the new bonds introduce also a stress on the subsurface atoms. The trade-off between these two effects [electronic (stabilizing) and elastic (destabilizing)] decides the stability of a surface reconstruction.

Looking at Fig. 3 we see that different As chemical potentials just shift the calculated $\gamma$ to higher energies and change the relative stability of the vicinal surfaces. The deviation of the projected (or reduced) surface energies as a function of the miscut angle from a straight line is due to the 
TABLE I. Step parameters for the $a, b, c, d, e, f$, and $g$ A and B steps. $\epsilon$ is the step energy entering Eq. (2) evaluated at different $\Delta \mu_{\mathrm{As}}$ values. $q$ are the fitted values (from the $a b$ initio calculated values; see text) of the step-step interaction entering Eq. (2). $L_{\text {step }}$ is the step length along the [110] direction, defined as the smallest length between two $\beta_{2}$ terraces having different structural motifs from the $\beta_{2}$ structure. $\Delta n_{\text {step }}^{i}$ is the step stoichiometry (number of As versus Ga atoms) relative to that of the $\beta_{2}$ surface, $N_{\mathrm{DB}}$ is the number of additional dangling bonds introduced with the steps, and $Q$ is the excess charge, that is the charge not transferred from the Ga to the As dangling bonds. $Q=0$ means the ECR is satisfied (complete transfer, all Ga DBs empty, and all As DBs completely full). $p_{x}$ and $p_{z}$ are the electrostatic dipole components per step unit length calculated as explained in the text minus those of the $\beta_{2}$ surface. $K_{e s}$ and $K_{e l}$ are the estimated electrostatic and elastic $K$ constants, respectively, of the $L^{-2}$ step-step interaction.

\begin{tabular}{|c|c|c|c|c|c|c|c|}
\hline \multicolumn{8}{|c|}{ Steps A } \\
\hline & $a$ & $b$ & $c$ & $d$ & $e$ & $f$ & $g$ \\
\hline$\epsilon\left(\Delta \mu_{\mathrm{As}}=0\right)(\mathrm{meV} / \AA)$ & -2.2 & 21.6 & 38.2 & 38.6 & 16.0 & 198.4 & 181.5 \\
\hline$\epsilon\left(\Delta \mu_{\mathrm{As}}=-0.32\right)(\mathrm{meV} / \AA)$ & -12.3 & 31.7 & 48.2 & 68.9 & 46.2 & 168.1 & 131.0 \\
\hline$\epsilon\left(\Delta \mu_{\mathrm{As}}=-0.58\right)(\mathrm{meV} / \AA)$ & -20.5 & 39.9 & 56.4 & 93.4 & 70.8 & 143.5 & 90.0 \\
\hline$q\left(\mathrm{meV} / \AA^{2}\right)$ & +864.0 & -154.24 & -149.52 & -59.39 & -83.6 & +3342.85 & +1531.52 \\
\hline$L_{\text {step }}(a)$ & 1.5 & 2.5 & 2.5 & 3.5 & 3.5 & 4.5 & 3.5 \\
\hline$\Delta n_{\text {step }}^{i}$ & +0.25 & -0.25 & -0.25 & -0.75 & -0.75 & +0.75 & +1.25 \\
\hline$N_{\mathrm{DB}}$ & 4 & 6 & 6 & 8 & 8 & 12 & 10 \\
\hline$Q(e)$ & -0.5 & +0.5 & +0.5 & +1.5 & +1.5 & -1.5 & -2.5 \\
\hline$p_{x}(e)$ & -0.20 & +0.14 & +0.13 & +0.52 & +0.53 & -0.14 & -0.45 \\
\hline$p_{z}(e)$ & 0.06 & 0.06 & 0.06 & +0.13 & +0.13 & +0.22 & +0.02 \\
\hline$K_{e s}(\mathrm{meV} \mathrm{\AA})$ & -82.58 & -38.32 & -32.46 & -566.93 & -589.71 & +14.05 & -438.89 \\
\hline$K_{e l}(\mathrm{meV} \mathrm{\AA})$ & +41.83 & & & & +322.37 & & \\
\hline \multicolumn{8}{|c|}{ Steps B } \\
\hline & $a$ & $b$ & $c$ & $d$ & $e$ & $f$ & $g$ \\
\hline$\overline{\epsilon\left(\Delta \mu_{\mathrm{As}}=0\right)(\mathrm{meV} / \AA)}$ & 137.91 & 162.44 & 140.47 & 138.79 & 134.14 & 132.35 & 124.13 \\
\hline
\end{tabular}

step-step interaction $q$ that does not change with $\Delta \mu_{\mathrm{As}}$. Indeed, by combining Eq. (1) with Eq. (2) we obtain for $\epsilon_{i}\left(\mu_{\mathrm{As}}\right)$ a linear dependence on the As chemical potential:

$$
\epsilon_{i}\left(\Delta \mu_{\mathrm{As}}\right)=\epsilon_{i}\left(\Delta \mu_{\mathrm{As}}=0\right)+\frac{\Delta n_{\mathrm{step}}^{i}}{L_{\perp}} \Delta \mu_{\mathrm{As}},
$$

where $L_{\perp}=2 a(4 a)$ is the lateral dimension of the A (B) steps surface unit cells. In Table I we report the main parameters characterizing the structure of A steps: the step length $L_{\text {step}}$, the step stoichiometry $\Delta n_{\text {step }}^{i}$, and the additional number of dangling bonds $N_{\mathrm{DB}}$. Equation (3) stresses that the step relative stability depends on the growth conditions.

\section{B. Step-step interaction}

From Fig. 3 we can see that B steps are much less interacting than A steps in agreement with the experiment of Lelarge et al. [15] mentioned in the Introduction. Indeed, we find in most cases an almost straight dependence of the surface energy on the miscut angle. However, the step-step interaction parameter $q$ extracted using Eq. (2) is very sensitive to the number of calculated values and to small details of the $\gamma$ versus $\alpha$ curves. To test the sensitivity of the value of $q$ on the details of the fitted curves we show in Fig. 4 other fits where one calculated point was omitted: the one corresponding to the largest $\alpha$ for which the point dipole model should not work well or, alternatively, the $\gamma\left(0, \mu_{\mathrm{As}}\right)$ final point corresponding to an infinite distance between the steps, because of the possible error in the alignment of the step surface energies with the $\beta_{2}$ surface energy (estimated within $0.05 \mathrm{meV} / \AA^{2}$ ). We can see that the extrapolated $\beta_{2}$ value in the case of step $\mathrm{A} a$ is in agreement with the value obtained using all the calculated values, while for step $\mathrm{A} e$ we obtain for the extrapolated $\gamma\left(0, \mu_{\mathrm{As}}\right)$ a value out of the range of the estimated alignment error. We can see from this test that the numerical value of $q$ is very sensitive to small changes of the $\gamma$ versus $\alpha$ curvature. The calculations show also that at least four calculated points are necessary to obtain a consistent estimate of the step-step interaction parameter $q$. However, the fourth point (calculated for the step A $a$ structure but not for the step A $e$ structure) corresponds to steps separated by four $\beta_{2}$ unit cells. This amounts to very large unit cells (500 atoms for the calculated fourth point of the A $a$ vicinal surface having a smaller unit cell size). Unfortunately we are unable to provide an equally accurate value for the larger $\mathrm{A} e$ vicinal surface with a four $\beta_{2}$ long terrace using our computational tool and choice of parameters (energy cutoff, $k$-point grids, norm-conserving pseudopotentials, etc.).

The $q$ parameter appearing in Eq. (2) is related to the $K$ constant of the $L^{-2}$ step-step interaction by $q=K / L_{\perp}^{3}$. For $\mathrm{A} a$ and $\mathrm{A} e$ steps we obtain $q_{a}=864 \mathrm{meV} / \AA^{2}$ and $q_{e}=$ $-84 \mathrm{meV} / \AA^{2}$, that is steps $a$ have a repulsive interaction and steps $e$ interact attractively. In the same way the calculations hint to a repulsive interaction for steps $\mathrm{A} f$ and $\mathrm{A} g$ and to an attractive interaction for steps $\mathrm{A} b, \mathrm{~A} c$, and $\mathrm{A} d$, that is the step-step interaction is weakly attractive for the As-richer steps (acceptors $Q>0$ ) and strongly repulsive for the As-poorer steps (donors $Q<0$ ).

The important issue here is that some steps seem to interact attractively contrary to the predictions of the elastic force-dipole model. Indeed, the elastic line point force dipole components $F_{i}=A_{i} \frac{d(\delta(\vec{r}))}{d x}, i=x, z$ ( $\delta$ is the Dirac 

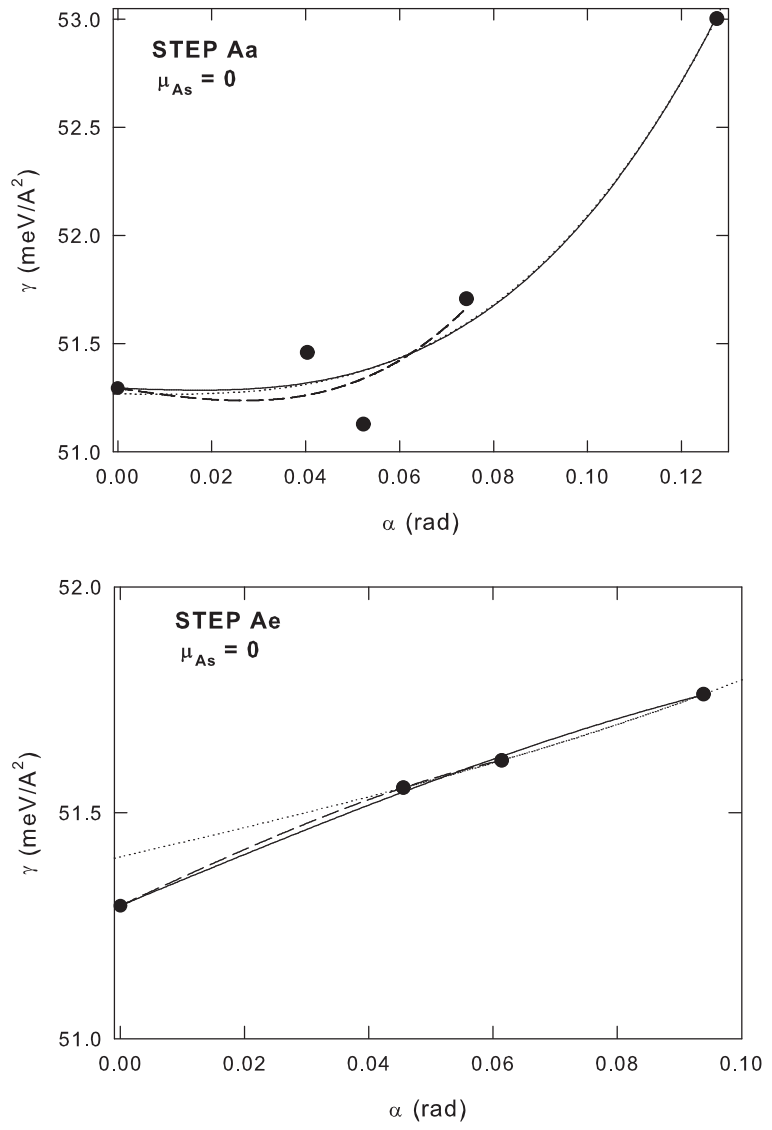

FIG. 4. Above: step A $a$. Solid line: all five values, $\epsilon=$ $-2.2 \mathrm{meV} / \AA, q=+864.0 \mathrm{meV} / \AA^{2}$; dashed line: four values including $\gamma_{\beta_{2}}, \epsilon=-9.0 \mathrm{meV} / \AA, q=+1481 \mathrm{meV} / \AA^{2}$; dotted line: four values without $\gamma_{\beta_{2}}, \epsilon=-0.8 \mathrm{meV} / \AA ⿻, q=+845 \mathrm{meV} / \AA^{2}$, predicted $\gamma_{\beta_{2}}+51.27 \mathrm{meV} / \AA^{2}$, calculated $51.29 \mathrm{meV} / \AA^{2}$. Below: step $\mathrm{A} e$. Solid line: all four values, $\epsilon=16.0 \mathrm{meV} / \AA, q=-83.6$ $\mathrm{meV} / \AA^{2}$; dashed line: three values including $\gamma_{\beta_{2}}, \epsilon=17.8 \mathrm{meV} / \AA$, $q=-291.6 \mathrm{meV} / \AA^{2}$; dotted line: three values without $\gamma_{\beta_{2}}, \epsilon=$ $9.0 \mathrm{meV} / \AA, q=+69.2 \mathrm{meV} / \AA^{2}$, predicted $\gamma_{\beta_{2}}+51.40 \mathrm{meV} / \AA^{2}$, calculated $51.29 \mathrm{meV} / \AA^{2}$.

$\delta$ function), located at the step line $x$ (the direction orthogonal to the step direction, in our case $x=[110]$ ) can be shown to generate the displacements [20]:

$$
u_{x}(x)=\frac{2\left(v^{2}-1\right)}{\pi E} \frac{A_{x}}{x}, \quad u_{z}(x)=\frac{2\left(v^{2}-1\right)}{\pi E} \frac{A_{z}}{x} .
$$

$E=85.5 \mathrm{GPa}$ is GaAs Young modulus, $v=0.31$ is the GaAs Poisson ratio, and $A_{i}$ are the components of the force dipoles. The elastic energy is given by

$$
W_{e l}^{\mathrm{tot}}=\frac{1}{2} \sum_{n, m}\left[\int d x\left[F_{x}^{n}(x) u_{x}^{m}(x)+F_{z}^{n}(x) u_{z}^{m}(x)\right]\right],
$$

where $n$ and $m$ are the step indexes. From these expressions we can see that the elastic step self-energy (sum of the terms with $n=m$ ) is always positive since the force and displacement fields are equally oriented. The terms with $n \neq m$ constitute the elastic step interaction. For like-oriented steps the energy is also positive since forces and displacements on the different steps distanced by $L$ are similarly oriented and the elastic step interaction energy is given by

$$
W_{e l}=\frac{\pi\left(1-v^{2}\right)}{3 E} \frac{A^{2}}{L^{2}}=\frac{K_{e l}}{L^{2}} .
$$

For opposite oriented steps on the contrary the elastic interaction energy predicted by the model is attractive. It has been shown in the literature that for like-oriented steps on stress-free surfaces the elastic interaction remains repulsive even when orders beyond the dipolar one or better models of the force fields at steps are considered [20]. Obviously these extensions of the model, although incapable to change the sign of the step interaction, introduce further unknown parameters. Attractive interactions however were inferred experimentally by the STM analysis of the step surface distributions on $\mathrm{Cu}(001)$ [25]. On the theoretical point of view an elastic attractive behavior between steps was shown to arise only in the case of a vicinal surface subjected to an external stress such as that induced by the growth on a mismatched substrate [26]. The problem of the possible origin of the step attractive interactions has been largely debated in the literature [27] where many different speculations have been proposed but not much progress has been done since. At $T=0 \mathrm{~K}$ the only other possible contribution to the dipolar step interaction has an electrostatic nature. This has been inferred in the literature [28] whenever the elastic point dipole model was unable to fit the data points. An explicit account of the electrostatic contribution was given to model the step energy on II-VI (001) surfaces [29], thus explaining the surface island shapes, but the step interaction contribution having the dipolar form $K / L^{2}$ was never proposed. We derive here the expression of a dipolar electrostatic step interaction following the same assumptions made for the derivation of the MP elastic point dipole interaction.

\section{Electrostatic dipole model and electrostatic step interaction}

We derive the interaction energy of an infinite sequence of line electrostatic dipoles. We consider a linear density of point electrostatic dipoles located at the step line (i.e., at $x=0)$. Differently from the elastic interactions the line dipoles $p$ interact both through the material and through the vacuum, and the electric field components depending on $p_{z}$ are discontinuous at the surface. After integration along the step line ( $y$ direction) one finds the expression for the electric fields at the surface $z=0$ as

$$
\begin{aligned}
& E_{x}(x)=+k \frac{4 p_{x}}{\left(1+\epsilon_{r}\right) x^{2}} \\
& E_{z}(x)=-k \frac{4 p_{z}}{\epsilon_{r}\left(1+\epsilon_{r}\right) x^{2}} \quad \text { material, } \\
& E_{z}(x)=-k \frac{4 p_{z}}{\left(1+\epsilon_{r}\right) x^{2}} \quad \text { vacuum, }
\end{aligned}
$$

where $k$ is the vacuum electrostatic constant, $p$ is the dipole linear density, and $\epsilon_{r}=12.9$ the GaAs relative dielectric constant of the material. This expression is equivalent to the expression reported for the displacement field under the point force dipole, Eq. (4), at the step location $x=0$ [20]. 
The electrostatic interaction energy is then calculated as

$$
W_{e s}=-\frac{1}{2} \sum_{n, m \neq n}\left[\int d x\left[p_{x}^{n}(x) E_{x}^{m}(x)+p_{z}^{n}(x) E_{z}^{m}(x)\right]\right] .
$$

After integration we find that the electrostatic interaction energy of an infinite sequence of point dipole lines distanced by $L$ along $x$ is given by

$$
W_{e s}=k \frac{\pi\left[-2 \epsilon_{r} p_{x}^{2}+\left(1+\epsilon_{r}\right) p_{z}^{2}\right]}{3 \epsilon_{r}\left(1+\epsilon_{r}\right)} \frac{1}{L^{2}}=\frac{K_{e s}}{L^{2}},
$$

where we have taken the average value of the electrostatic field across the vacuum and the material. From these expressions we see that, while the dipole elastic interactions between likeoriented steps are always repulsive, see Eq. (6), the electrostatic ones can be in principle both repulsive or attractive. To assess what the situation is for steps on $\operatorname{GaAs}(001)$ we use a point charge model based on the electron counting rule (ECR) [30,31]. Following the ECR, III-V surfaces stabilize through a charge transfer from the DBs on Ga atoms, lying at higher energies, to the DBs on the As atoms, lying at lower energies. For the octet rule each Ga DB has a $0.75 e$ charge, while each As DB has a $1.5 e$ charge when bonded to two Ga and one As atom in a dimer. If the number of both kinds of DBs is right all $\mathrm{Ga}$ DBs become empty while As DBs become fully occupied with two electrons. In this case the ECR is said to be satisfied: the surface is semiconductor and the system lowers considerably its energy. This is the case of the $\beta_{2}(2 \times 4)$ surface. Following the charge transfer the undercoordinated surface $\mathrm{Ga}$ (As) atoms become positively (negatively) charged with $q_{\mathrm{Ga}}=+3 / 4 e$ $\left(q_{\text {As }}=-0.5 e\right)$. The stepped surfaces do not satisfy the ECR: steps, like $\mathrm{A} a, \mathrm{~A} f$, and $\mathrm{A} g$, have an excess of Ga DBs; thus a charge $Q$ cannot be transferred to As DBs and we assume it remains localized in the original Ga DBs. Since the Ga DB state energies are closer to or within the conduction band these steps behave as donor defects [32]. This situation is indicated in Table I with $Q<0$. Steps $\mathrm{A} b, \mathrm{~A} c, \mathrm{~A} d$, and $\mathrm{A} e$ have instead an excess of As DBs; thus the As DBs remain still partially occupied after all the charge available from the Ga DBs has been transferred to them. The steps are acceptors and $Q>0$. This model of charge transfer allows us to ascribe point charges to the undercoordinated atoms at the surface. On the $\beta_{2}(2 \times 4)$ reconstructed terraces the Ga point charges are $+0.75 e$ and the As point charges are $-0.5 e$ [33]. Using these values and the calculated equilibrium atom positions we can calculate the step dipoles as $\vec{p}=\sum_{i} q_{i} \vec{r}_{i} / L_{\perp}$. The dipoles depend on how the step charges are distributed. We report here as an example the case where the untransferred charges remain localized at the DBs at the step making the corresponding ions less positive or negative than the $\beta_{2}$ 's. A localized charge arrangement of this kind is shown in Fig. 5 and the corresponding dipoles (subtracting the $\beta_{2}$ ones: $p_{x}=0$ and $p_{z}=-0.33$ ) are given in Table I together with the electrostatic energy constant $K_{e s}$ of the $L^{-2}$ dipole interaction. We have found that for most charge arrangements, even more delocalized, the dipole-dipole electrostatic energy is indeed negative; that is, the electrostatic dipole interaction for monolayer high steps tends to be attractive.

We find that, interestingly, the calculated $\vec{p}$ do not depend substantially on the terrace length $L$ between steps, which shows that the atom positions (and displacements) at step $i$ (we used the $a b$ initio calculated atom positions) are similar and independent of $L$. $\beta 2$

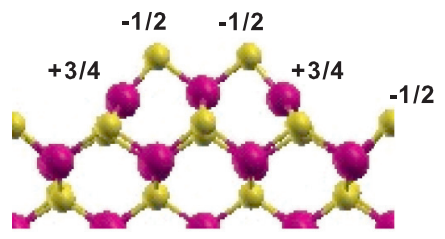

a
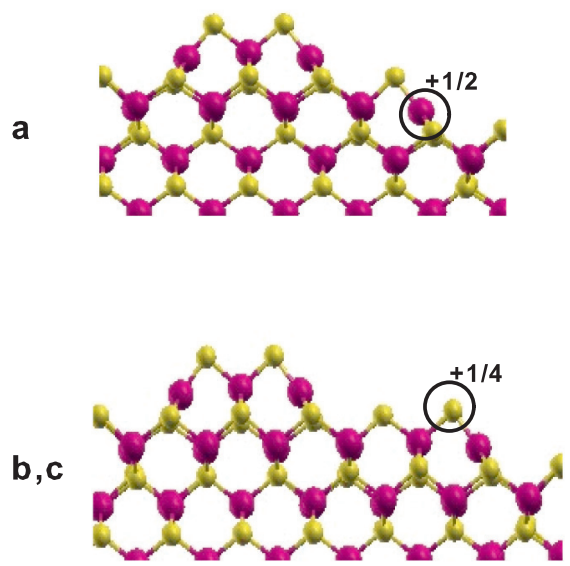

d,e

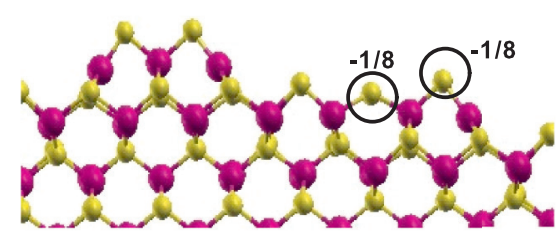

f

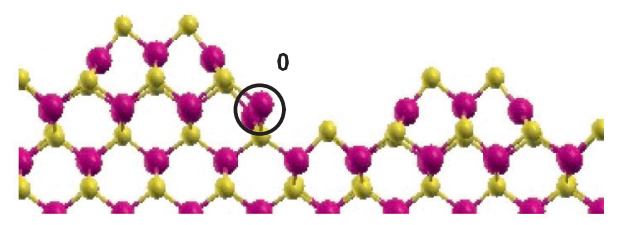

g

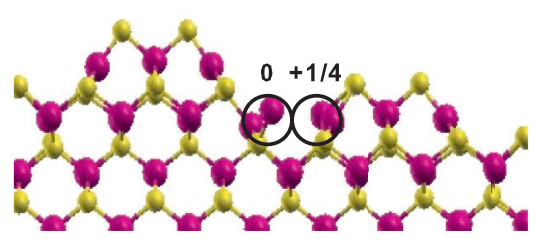

FIG. 5. (Color online) Point charge distribution used to calculate step point dipoles. In the step structures the charges assigned to the dangling bonds on the $\beta_{2}$ terrace are the same as for the $\beta_{2}$ structure. Yellow balls: arsenic atoms; purple balls: gallium atoms. 


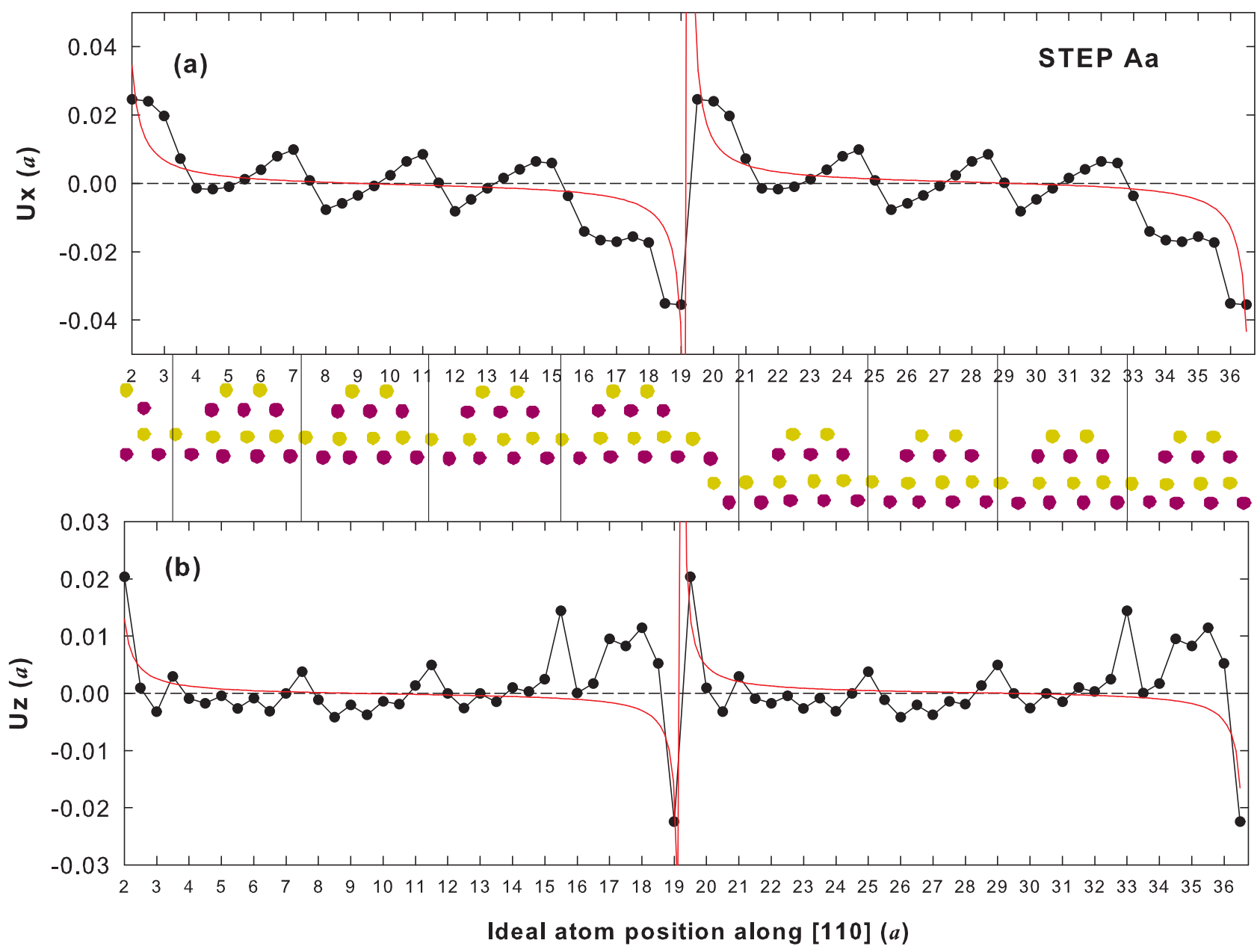

FIG. 6. (Color online) Red line: fit to the calculated atomic dispacements of step A $a$ (dots and solid line). Obtained values for the force dipole components are $A_{x}=-143.7 \mathrm{meV} / \AA$ and $A_{z}=-54.2 \mathrm{meV} / \AA$. (a) $U_{x}$ at atom positions $n a$ along [110]. (b) $U_{z}$ at atom positions $n a$ along [110]. In the middle is a ball and stick side view along [110] of the first few atomic planes; yellow dots: As atoms; purple dots: Ga atoms. The distance $L$ between steps if four $\beta_{2}$ unit cells.

\section{Elastic step interaction}

In Table I we give an estimate of the analogous elastic energy constant $K_{e l}$ within the elastic point dipole model for the steps $\mathrm{A} a$ and $\mathrm{A} e$. The force dipole $A_{x}$ and $A_{z}$ components entering Eq. (6) are extracted by the displacements (relative to those of the $\beta_{2}$ flat surface) of the atoms of the first six layers for the steps having smaller miscut angles by fitting these displacements to those given by the elastic dipole model Eq. (4).

We have summed the displacements over the atoms of the first six layers located at $x$ :

$$
U_{k}(x)=\sum_{i} u_{k}^{i}(x),
$$

where $u_{k}^{i}(x)$ are the $k=x, z$ components of atom $i$ displacement at $x$ along the [110] direction. This procedure allows us to obtain the behavior of a "continuum" surface layer comparable with the dipole model. The atom displacements at $x$ of the atoms belonging to the layers below the sixth have a negligible effect on the sum. The $U_{x}$ and $U_{z}$ displacements so obtained are shown in Figs. 6 and 7. We can see from the figures that even for surfaces having complex reconstructions and a short distance between steps the obtained displacements
$U_{x}$ follow approximately a dipolar behavior at the step. The $U$ curves go to zero in the region between the steps as required by the dipole model. Interestingly, we can see that there are oscillations in the sign of the displacements overimposed on the dipolar behavior with the periodicity of the surface reconstruction features. In particular, we can see that larger displacements (step $\mathrm{A} e$ ) are generated by a larger number of As dimers at the step (the displacements relative to step $\mathrm{A} b$ not shown have values falling between those of steps $\mathrm{A} a$ and $\mathrm{A} e$ ).

This result translates in weaker force dipole components $A$ for step A $a$ than for step A $e$. We obtain a similar result also in the case of the shortest distance between the steps. The $U_{i}$ are larger for step A $e$ than for step A $a$. Within the predictions of the dipole model the repulsive elastic contribution to the step energy should be smaller for step A $a$ than for step A $e$. Since the same force components $A_{i}$ enter also the expression of the elastic contribution to the step energy $\epsilon$ this implies a smaller elastic step energy for step A $a$ which would be in agreement with the larger stability we have found with the $a b$ initio calculations. However, our $a b$ initio calculations found also a strong repulsive interaction at short distances for step $\mathrm{A} a$ which is contrary to the elastic dipole model predictions. 


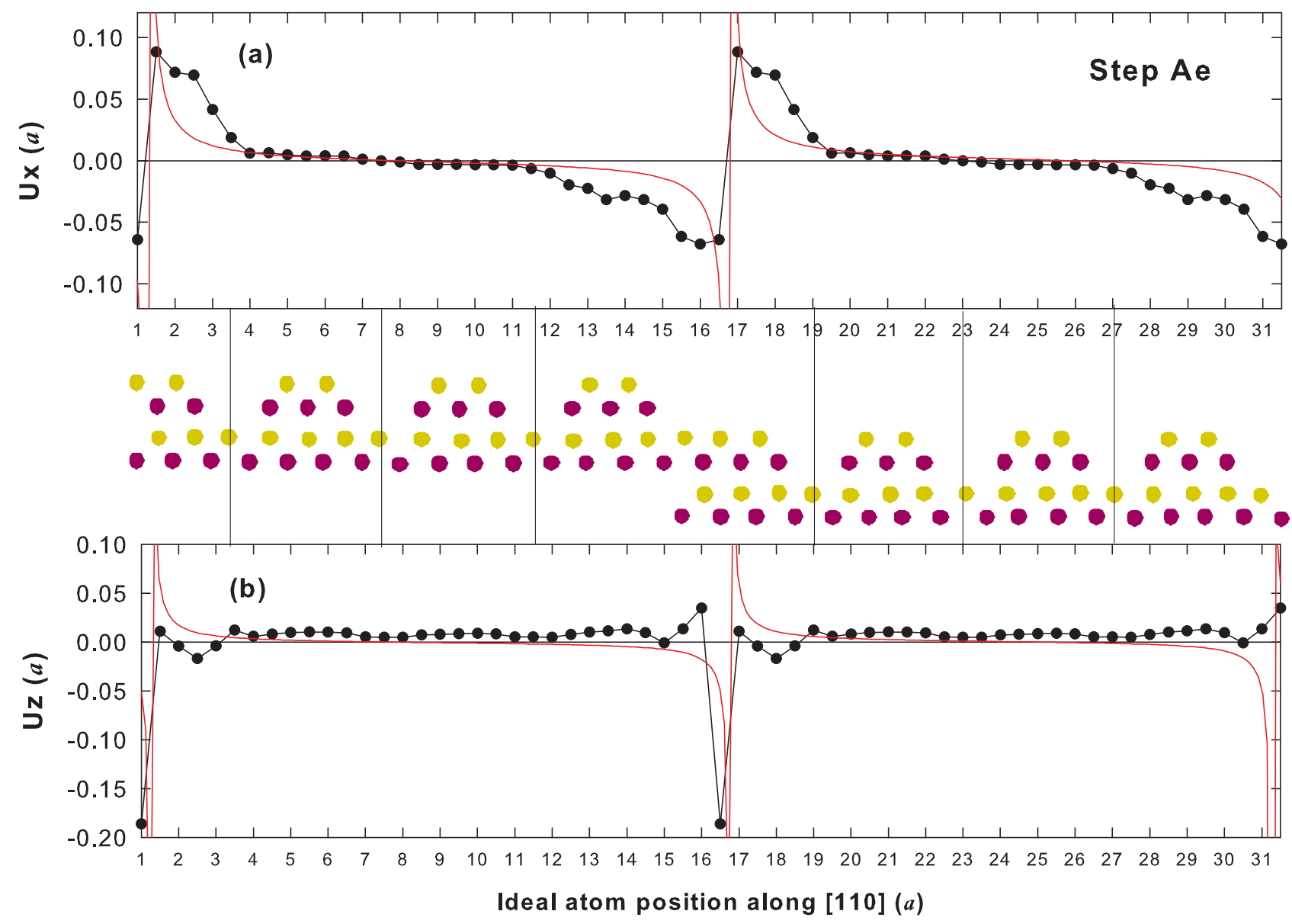

FIG. 7. (Color online) Red line: fit to the calculated atomic displacements of step Ae (dots and solid line). Obtained values for the force dipole components are $A_{x}=-377.3 \mathrm{meV} / \AA$ and $A_{z}=-198.4 \mathrm{meV} / \AA$. (a) $U_{x}$ at atom positions $n a$ along [110]. (b) $U_{z}$ at atom positions $n a$ along [110]. In the middle is a ball and stick side view along [110] of the first few atomic planes; yellow dots: As atoms; purple dots: Ga atoms. The distance $L$ between steps is three $\beta_{2}$ unit cells.

Summarizing our results we find that the (elastic and electrostatic) dipole model predicts weaker dipolar step interactions between the Ga-rich steps (like step $\mathrm{A} a$ ) than for the As-rich steps (like step A $e$ ).

\section{DISCUSSION AND CONCLUSIONS}

In this paper we report on direct $a b$ initio calculations to study the structural properties of steps on the $\mathrm{GaAs}(001)$ surface reconstructed $\beta_{2}(2 \times 4)$. The calculated surface energies of vicinal surfaces featuring different step structures and orientations have been compared to the standard elastic dipole model of Marchenko and Parshin [12]. In this model the action of steps on the flat surface is modeled via lines of point force dipoles which mutually interact with long range elastic interactions. The resulting elastic energy was shown to scale with the distance $L$ between steps as $K L^{-2}$. The fit of the $a b$ initio calculated surface energies to the model allows us to extract the two parameters describing the step properties: the step energy $\epsilon$ and the step-step interaction $q$. The comparison reveals that some step structures interact attractively contrary to the dipole model of the elastic interaction which predicts that between like-oriented steps the interaction is repulsive. The elastic dipole model was found to be better applicable to steps whose distances are more than a few lattice parameters apart. For instance in the case of fcc metal surfaces $(\mathrm{Ag}$, $\mathrm{Au}, \mathrm{Cu}, \mathrm{Pd}$, and $\mathrm{Pt}$ ) the elastic dipole model was able to fit the semiempirically calculated values for step distances larger than $3 a_{0}$ [28]. The authors of that paper found also that adding an attractive $L^{-3}$ term improved the fit over all distances. The continuum theory is indeed expected to fail at very short step distances where the discreteness of the atomic lattice becomes important. In that paper, as in a large part of the following literature, the topic of the origin of attractive interactions has been debated but not fully understood.

We first have tackled the problem of a possible attractive interaction within the dipole interaction model recognizing that relevant charge transfer at the step can create electrostatic dipoles. Likewise for the elastic dipole interaction we have considered the interaction between lines of point electrostatic dipoles obtaining the expression for the electrostatic interaction energy scaling as $L^{-2}$ with the step distance. The estimates of this expression using concepts from the electron counting rule have enabled us to show that some step structures indeed interact attractively. Our estimates show that the elastic and electrostatic dipole interactions have a similar magnitude. The sign of the final resulting dipole interaction thus comes out 
from the interplay between the elastic contribution (always repulsive) and the electrostatic contribution (attractive or repulsive) that depend ultimately on the specific step structure.

This analysis however does not explain the ab initio results relative to the fact that "donor" steps have a strong repulsive interaction, while "acceptor" steps have a weaker attractive interaction. The elastic and electrostatic dipole model does not explain our calculated $q$, since it predicts elastic repulsive interactions weaker for the donor $\mathrm{A} a$ step than for the acceptor A $e$ step, contrary to the $a b$ initio results, and electrostatic attractive dipole interactions stronger for the A $e$ step than for the A $a$ step, in qualitative agreement with the $a b$ initio results.

We notice that in our calculatons the shortest steps are separated by one $\beta_{2}$ unit cell, i.e., $L=2.83 a_{0}$. This value of $L$ falls within a distance range where the applicability of the dipole interaction model is questionable. Thus we argue that at such short step distances a different kind of interaction becomes dominant. The observation that a repulsive behavior is associated to steps with an electronic "donorlike" band structure, while an attractive behavior is associated to steps with an electronic "acceptorlike" band structure suggests an quantum origin for the short distance step-step interaction which is accessible precisely to the $a b$ initio calculations that treat electronic and structural degrees of freedom on the same footing.

In the case $Q>0$ (acceptorlike step states) the system Fermi energy falls below the top of the valence band; thus the partially occupied step states have substantially a valence character. The opposite is true for the case $Q<0$ where the system Fermi level falls much higher in energy above the bottom of the conduction band. In this case the partially occupied step states lying at higher energies have a more conduction state character. In the case of short distances between steps it is possible that the step states with energies near the gap edges have a substantial overlap between them (and likely with the other surface states). Since usually this overlap is larger for the states at the bottom of the conduction band than for those at the top of the valence band (as testified, for example, by the larger band dispersion and consequently smaller effective masses of the states at the bottom of the conduction band than at the top of the valence band in most III-V semiconductors and the $\beta_{2}$ surface), we speculate that the donorlike step states interact repulsively more strongly than the acceptorlike states. This repulsive interaction would lead to a higher positive contribution to the total energy for the donor steps and, as a consequence, to a larger repulsive value for the step interaction $q$ term. To explain the repulsive interaction we observe that the total energy of the ground state can be written as

$$
E=\sum_{n}\left\langle\psi_{n}|\widehat{T}+\widehat{V}| \psi_{n}\right\rangle+E_{H}+E_{x c}+E_{\text {ion-ion }},
$$

where $\left|\psi_{n}\right\rangle$ are the occupied states. $\widehat{T}$ is the kinetic energy operator, $\widehat{V}$ is the one-body potential energy acting on the electrons, $E_{H}$ is the electron-electron Hartee energy, $E_{x c}$ is the electron-electron exchange-correlation energy, and $E_{\text {ion-ion }}$ is the ion-ion Ewald interaction energy. Let's assume that we can separate the contributions to the Hartree term:

$$
\frac{e^{2}}{2} \iint \frac{n(\vec{r}) n\left(\overrightarrow{r^{\prime}}\right)}{\mid \vec{r}-\overrightarrow{r^{\prime} \mid}} d \vec{r} d \overrightarrow{r^{\prime}}
$$

$[n(\vec{r})$ is the particle density at $\vec{r}]$ due uniquely to the step states. They would read as

$$
E_{H}^{\text {step }}=\frac{e^{2}}{2} \iint \frac{\left|\psi_{i}(\vec{r})\right|^{2}\left|\psi_{j}\left(\overrightarrow{r^{\prime}}\right)\right|^{2}}{\left|\vec{r}-\overrightarrow{r^{\prime}}\right|} d \vec{r} d \overrightarrow{r^{\prime}},
$$

where $|i\rangle$ and $|j\rangle$ are $\left|a_{i}\right\rangle$ and $\left|a_{j}\right\rangle$ step acceptor states on neighboring steps $i$ and $j$ (for instance, Wannier functions localized at the steps) or $\left|d_{i}\right\rangle$ and $\left|d_{j}\right\rangle$ the analogous donor states. From this equation we can see that the larger the step functions overlap in space, the more the electronic charge is evenly distributed over all the step and terrace region, leading to a higher contribution to the Hartree integral. Another way to look at the same concept is to observe that the sum of the single-particle eigenvalues $\epsilon_{i}$ (not to be confused with the step energies) is the largest electron contribution to the total energy $E$ if $E$ is expressed in the analogous alternative form:

$E=\sum_{i} \epsilon_{i}-E_{H}+\int\left(\epsilon_{x c}-V_{x c}\right) n(\vec{r}) d \vec{r}+E_{\text {ion-ion }}$,

where $\epsilon_{x c}$ and $V_{x c}$ are the exchange and correlation energy and potential, respectively. Now we can observe that, in the case of donor steps, the step-related levels lying within the conduction band are occupied since the Fermi level is within the conduction band, while the occupied levels related to the acceptor step states have lower energies since in this case the Fermi energy falls within the valence band. Thus the first term on the right side of Eq. (12) is larger for donor steps than for acceptor steps. This difference is larger when the step-related occupied states are not a negligible part of all the occupied states, that is, in the case of close by steps (smaller unit cells). Obviously this hypothesis needs further work to be fully understood.

Finally, monolayer steps on $(2 \times 4) / c(2 \times 8)$ vicinal Sidoped GaAs(001) surfaces ( $n$ type doping) have been visualized using ultrahigh-vacuum scanning tunneling microscopy [34]. The observed step structures correspond to the $\mathrm{A} b$ steps of Fig. 1 reported to be acceptors. We find these steps unstable (i.e., $\epsilon_{b}>0$ and $\epsilon_{b}>\epsilon_{a}$ at all $\Delta \mu_{\text {As }}$; see Table I); thus the probability to be formed at a given temperature $\mathrm{T}$ would be much lower than for step A $a$ (which instead has not yet been visualized to our knowledge). The explanation of the experimental observation of step $\mathrm{A} b$ could be given conjecturing that the dopant atoms lower the formation energy of steps $\mathrm{A} b$ and make them more likely to form. It was shown indeed [35] that dopant atoms of $n$ and $p$ types in semiconductor nanocrystals are preferentially located at the surface (where the strain they induce in the matrix can be more effectively relieved) close to one another because in this way a charge transfer occurs which leads to a considerable lowering of the system energy. The same behavior could be at work also in this case with the silicon $n$ dopant states interacting with the acceptorlike states of step $\mathrm{A} b$ in such a way as to lower the step $\mathrm{A} b$ formation energy. Other surface calculations have shown that a $Q<0$ situation (i.e., Fermi level above the bottom of 
the conduction band) can indeed stabilize acceptor surface defects [31].

Since the step energy $\epsilon$ depends on the As chemical potential it is also greatly influenced by the epitaxial growth conditions. Clearly, conditions of high temperature (increasing the probability of formation of the less stable steps) and a high As flux can increase the probability of formation of the As-rich steps.

These considerations lead us to speculate that by doping or through the choice of proper growth conditions one can impose what step structures can be formed and, as a consequence, manipulate the step-step interaction. On the other hand, it is known that attractive interactions between steps can lead to step bunching [26]; thus we could expect that when the conditions are such as to stabilize the As-rich steps we should assist to the formation of step bunchings. To confirm this expectation experimental works have indeed found that silicon doping [15] or a very high As to Ga flux ratio at high growth temperatures [16] lead to step bunching on $\mathrm{GaAs}(001)$, whereas step bunching never occurs at a low As to Ga flux in absence of doping [15]. Our calculations suggest that at the origin of the bunching behavior could be the stabilization, via $n$ doping or high temperatures and As fluxes, of acceptor steps which then interact attractively. These ideas open to the exciting prospect of step engineering via doping and/or appropriate changes in growth conditions.
[1] J. Stangl, V. Holý, and G. Bauer, Rev. Mod. Phys. 76, 725 (2004).

[2] E. Placidi, F. Arciprete, M. Fanfoni, F. Patella, E. Orsini, and A. Balzarotti, J. Phys.: Condens. Matter 19, 225006 (2007).

[3] H.-C. Jeong and E. D. Williams, Surf. Sci. Rep. 34, 171 (1999).

[4] D. J. Chadi, Phys. Rev. Lett. 59, 1691 (1987).

[5] O. L. Alerhand, D. Vanderbilt, R. D. Meade, and J. D. Joannopoulos, Phys. Rev. Lett. 61, 1973 (1988).

[6] O. L. Alerhand, A. N. Berker, J. D. Joannopoulos, D. Vanderbilt, R. J. Hamers, and J. E. Demuth, Phys. Rev. Lett. 64, 2406 (1990).

[7] T. W. Poon, S. Yip, P. S. Ho, and F. F. Abraham, Phys. Rev. Lett. 65, 2161 (1990).

[8] X.-P. Li, D. Vanderbilt, and R. D. King-Smith, Phys. Rev. B 50, 4637 (1994).

[9] W. G. Schmidt and J. Bernholc, Phys. Rev. B 61, 7604 (2000).

[10] R. Viswanathan, A. Madhukar, and S. Ogale, J. Cryst. Growth 150, 190 (1995).

[11] M. Rosini, P. Kratzer, and R. Magri, Phys. Status Solidi C 7, 181 (2010).

[12] V. I. Marchenko and A. Y. Parshin, Sov. Phys. JETP 52, 129 (1980).

[13] E. J. Heller, Z. Y. Zhang, and M. G. Lagally, Phys. Rev. Lett. 71, 743 (1993).

[14] E. J. Heller and M. G. Lagally, Appl. Phys. Lett. 60, 2675 (1992).

[15] F. Lelarge, Z. Z. Wang, A. Cavanna, F. Laruelle, and B. Etienne, EPL (Europhys. Lett.) 39, 97 (1997).

[16] F. Arciprete, E. Placidi, R. Magri, M. Fanfoni, A. Balzarotti, and F. Patella, ACS Nano 7, 3868 (2013).

[17] P. Giannozzi, S. Baroni, N. Bonini, M. Calandra, R. Car, C. Cavazzoni, D. Ceresoli, G. L. Chiarotti, M. Cococcioni, I. Dabo, A. Dal Corso, S. de Gironcoli, S. Fabris, G. Fratesi, R. Gebauer, U. Gerstmann, C. Gougoussis, A. Kokalj, M. Lazzeri, L. Martin-Samos, N. Marzari, F. Mauri,
R. Mazzarello, S. Paolini, A. Pasquarello, L. Paulatto, C. Sbraccia, S. Scandolo, G. Sclauzero, A. P. Seitsonen, A. Smogunov, P. Umari, and R. M. Wentzcovitch, J. Phys.: Condens. Matter 21, 395502 (2009).

[18] M. Rosini, R. Magri, and P. Kratzer, Phys. Rev. B 77, 165323 (2008).

[19] E. Gruber and W. Mullins, J. Phys. Chem. Solids 28, 875 (1967).

[20] P. Müller and A. Saúl, Surf. Sci. Rep. 54, 157 (2004).

[21] H. J. Zandvliet, Phys. Rep. 388, 1 (2003).

[22] C. Jayaprakash, C. Rottman, and W. F. Saam, Phys. Rev. B 30, 6549 (1984).

[23] M. Rosini and R. Magri, ACS Nano 4, 6021 (2010).

[24] N. Ghaderi, M. Peressi, N. Binggeli, and H. Akbarzadeh, Phys. Rev. B 81, 155311 (2010).

[25] J. Frohn, M. Giesen, M. Poensgen, J. F. Wolf, and H. Ibach, Phys. Rev. Lett. 67, 3543 (1991).

[26] J. Tersoff, Y. H. Phang, Z. Zhang, and M. G. Lagally, Phys. Rev. Lett. 75, 2730 (1995).

[27] A. C. Redfield and A. Zangwill, Phys. Rev. B 46, 4289 (1992).

[28] R. Najafabadi and D. Srolovitz, Surf. Sci. 317, 221 (1994).

[29] D. Martrou, J. Eymery, and N. Magnea, Phys. Rev. Lett. 83, 2366 (1999).

[30] M. D. Pashley, Phys. Rev. B 40, 10481 (1989).

[31] C. Hogan, R. Magri, and R. Del Sole, Phys. Rev. Lett. 104, 157402 (2010).

[32] C. Hogan, R. Magri, and R. Del Sole, Phys. Rev. B 83, 155421 (2011).

[33] S. B. Zhang and A. Zunger, Phys. Rev. B 53, 1343 (1996).

[34] K. Kanisawa, H. Yamaguchi, and Y. Horikoshi, Phys. Rev. B 54, 4428 (1996).

[35] F. Iori, E. Degoli, R. Magri, I. Marri, G. Cantele, D. Ninno, F. Trani, O. Pulci, and S. Ossicini, Phys. Rev. B 76, 085302 (2007). 